\title{
Reduction of Conducted Electromagnetic Emissions in Multi-Inverter Drive Systems
}

\author{
Mario Cacciato*, Alfio Consoli**, Giuseppe Scarcella**, Antonio Testa*** \\ D.I.E. - University of Rome "La Sapienza", Via Eudossiana, 18 -00184 Roma, ITALY * \\ D.E.E.S. - University of Catania Viale A.Doria, 6 - 95125 Catania, ITALY ** \\ D.F.M.T.F.A. - University of Messina Salita Sperone, $31-98166$ Messina, ITALY ***
}

Conducted emissions are caused by circulation of high frequency common mode currents generated by steep variations of the common mode voltage. In this paper, an inexpensive technique is presented that allows to noticeably reduce conducted electromagnetic emissions of multi-inverter drive systems by compensating common mode voltage variations.

Key words: EMI, common mode currents, pulsewidth modulation strategy.

\section{Introduction}

In modern electric drives, strong conducted Electro-Magnetic Emissions (EMI) are caused by circulation of common mode capacitive currents, that, although present in any electric drive, become relevant in last systems as a consequence of the increased rate of variation of the common mode voltage.

Limitation of conducted EMI is essential to increase the reliability and the electromagnetic compatibility of electric drives [1-3]. Such an issue becomes particularly critical in multi-inverter systems composed of several drives connected on the same main line. In such systems, largely present in industrial plants as parts of robots, servo systems and production lines, high level of conducted EMI are easily reached, due to the composition of the common mode currents generated by single inverters. In commercial electric drives, common mode current reduction is generally achieved by adding suitable filters at the inverter output, noticeably increasing costs and sizes. Alternatively, it is possible to act on the sequence of the inverter states using some specifically developed Pulse Width Modulation (PWM) strategies [4][5]. Such a techniques allow to effectively reduce common mode currents, but at the cost of a phase current distortion at high modulation indexes. A four legs inverter topology has been also proposed, that exploiting a suitable PWM strategy, able to keep constant the common mode voltage, thus theoretically eliminating common mode currents. However, such an approach requires an additional inverter leg, that increases the complexity of the system.

In the present paper, an inexpensive solution is proposed that allows to compensate the common mode voltage variation in multi-inverter drive systems without influencing the normal system operation at industrial frequencies. The proposed solution consists in a proper PWM strategy and in an high frequency connection of inverters by mean of high-pass filters. The high frequency connection allows to consider any two motor drive as a 6 wire system and any three motor drive as a 9 wire system at the high frequencies of the conducted emission. The PWM strategy selects the state sequences for each inverter and synchronizes the switching times, therefore, the common mode voltage of the global system is kept constant and no common mode currents ideally flow outside the drives along the ground and the mains. The low frequency voltage reference vectors of each motor are controlled independently. The suggested technique is developed in this paper for multi-inverter systems with two and three induction motor drives, but it can be applied to any multi-inverter ac motor system by suitably partitioning the drive set.

\section{Common mode currents in multi-inverter systems}

Common mode currents in electrical drive are generated from variations of the common mode voltage $v_{c m}$, defined as the potential between the neutral point of stator windings and the ground. In a $\mathrm{Y}$ connected three phase electric machine $v_{c m}$ is given by:

$$
v_{c m}=\frac{v_{M 1}+v_{M 2}+v_{M 3}}{3}
$$

where $v_{M l}, v_{M 2}$ and $v_{M 3}$ are the phase voltages. Common mode voltage variations can be determined from Tab. I according to the inverter switch state configuration.

TABLE I - INVERTER STATES AND COMMON MODE VOLTAGE

\begin{tabular}{|c|c|c|c|c|}
\hline STATE & $v_{M I}$ & $v_{M / 2}$ & $v_{M 3}$ & $\mathcal{V}_{c m}$ \\
\hline$V_{0}(0,0,0)$ & $-V_{D C} / 2$ & $-V_{D C} / 2$ & $-V_{D C}^{\prime} / 2$ & $-V_{D C} / 2$ \\
\hline$V_{1}(l, 0,0)$ & $V_{D C} / 2$ & $-V_{D C} / 2$ & $-V_{D C} / 2$ & $-V_{D C} / 6$ \\
\hline$V_{2}(1,1,0)$ & $V_{D C} / 2$ & $V_{D C} / 2$ & $-V_{D C} / 2$ & $V_{D C} / 6$ \\
\hline$V_{3}(0,1,0)$ & $-V_{D C} / 2$ & $V_{D C} / 2$ & $-V_{D C} / 2$ & $-V_{D C} / 6$ \\
\hline$V_{+}(0,1,1)$ & $-V_{D C} / 2$ & $V_{D C} / 2$ & $V_{D C} / 2$ & $V_{D C} / 6$ \\
\hline$V_{s}(0,0,1)$ & $-V_{D d} / 2$ & $-V_{D C} / 2$ & $V_{D C} / 2$ & $-V_{D d} 6$ \\
\hline$V_{6}(1,0,1)$ & $V_{D C} / 2$ & $-V_{D d} / 2$ & $V_{D C} / 2$ & $V_{D C} 6$ \\
\hline$V_{7}(1,1,1)$ & $V_{D C} / 2$ & $v_{D C} / 2$ & $V_{D C} / 2$ & $V_{D C}+2$ \\
\hline
\end{tabular}

An essentially capacitive common mode current path is normally present in any motor drive. As a result, steep variations of the common mode voltage generate common mode currents, that, circulating through the motor case ground connection, the earth and the main, reenter the power converter through the main connection. In a multi-drive system, the total common mode current flowing through the ground and the main is the sum of the common mode currents generated by each single drive.

The proposed idea is to connect the inverters by mean of three phase RLC filters, and to manage the sequence of the inverter states in order to maintain constant the global common mode voltage of the system. In the following, the proposed approach will be developed for two and three inverter systems. Thus, by suitably grouping the inverters it is possible to apply. the proposed approach to a system composed of any number of inverters 


\section{The Proposed Approach}

As the common mode current path behaves as a high pass filter, common mode currents can be avoided by holding constant the common mode voltage. Unfortunately, in a single three phase inverter drive the common mode voltage cannot be kept constant without generating stator current distortions. On the contrary, by suitably connecting a multi-inverter system it is possible to manage the sequence of the inverter states in order to obtain the correct phase voltages and a constant global common mode voltage.

A system composed of any even number of inverters can be partitioned in a set of two inverter subsystems. Instead, systems composed of an odd number of inverters can be partitioned in a set of two inverter subsystems, plus a single three inverter subsystem. Consequently, by solving the problem of keeping constant the global common mode voltage in two and three inverter systems, it is possible to deal with system of any number of inverters.

The proposed approach is firstly developed for a two drive system. Common mode voltages of two drives connected to the same main line are given by:

$$
\begin{aligned}
& v_{c m}^{\prime}=\frac{v_{M 1}^{\prime}+v_{M 2}^{\prime}+v_{M 3}^{\prime}}{3} \\
& v_{c m}^{\prime \prime}=\frac{v_{M 1}^{\prime \prime}+v_{M 2}^{\prime \prime}+v_{M 3}^{\prime \prime}}{3}
\end{aligned}
$$

Interconnecting the two inverters by the three phase RLC filters, as it is shown in Fig. 1, a six wire system is obtained, whose common mode voltage is given by:

$$
v_{c m}=v_{c m}^{\prime}+v_{c m}^{\prime \prime}
$$

then, selecting $v_{c m}$ to zero:

$$
v_{c m}^{\prime}=-v_{c m}^{\prime \prime}
$$

By fulfilling condition (5) common mode currents are forced to flow inside the two inverters, through the RLC filters, without influencing the machines, neither the ground, nor the main. Conducted common mode electromagnetic emissions and ground currents are consequently avoided. To accomplish condition (5), a suitable PWM technique has been developed based on the Space Vector Modulation (SVM) approach.

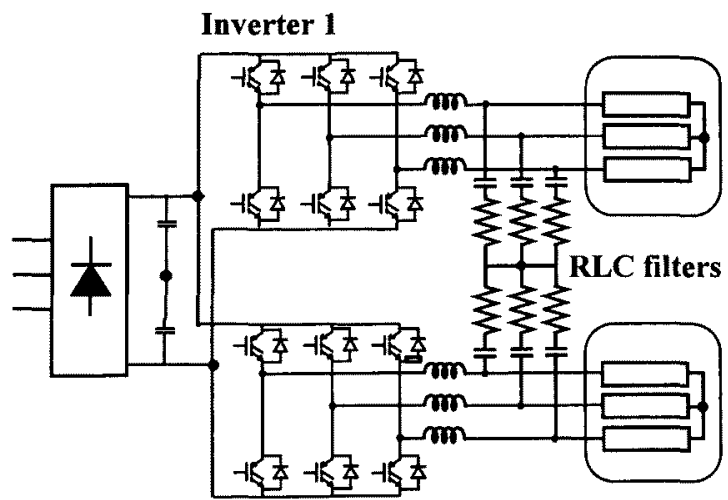

Inverter 2

Fig. 1 - RLC interconnection filters.
As shown in Fig. 2 and according to the proposed PWM strategy, the reference voltage vector is synthesized by mean of four inverter states, represented in the space vector plane by vectors $\bar{X}, \bar{Y}, \bar{Z}$ and $\bar{W}$. As shown in Fig. 3, the switching period is halved into two equal time segments. Inside the first time segment voltage modulation is performed by using only two odd states $\bar{X}_{1}$ and $\bar{Y}_{1}$ in one inverter and two even states $\bar{Z}_{2}$ and $\bar{W}_{2}$ in the other.
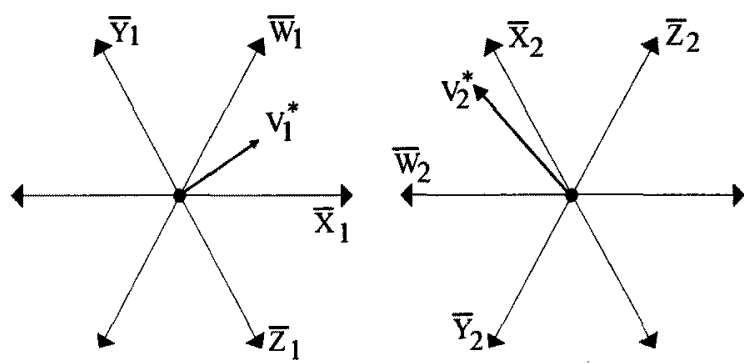

Fig. 2 - Space vector plane for the two inverters.

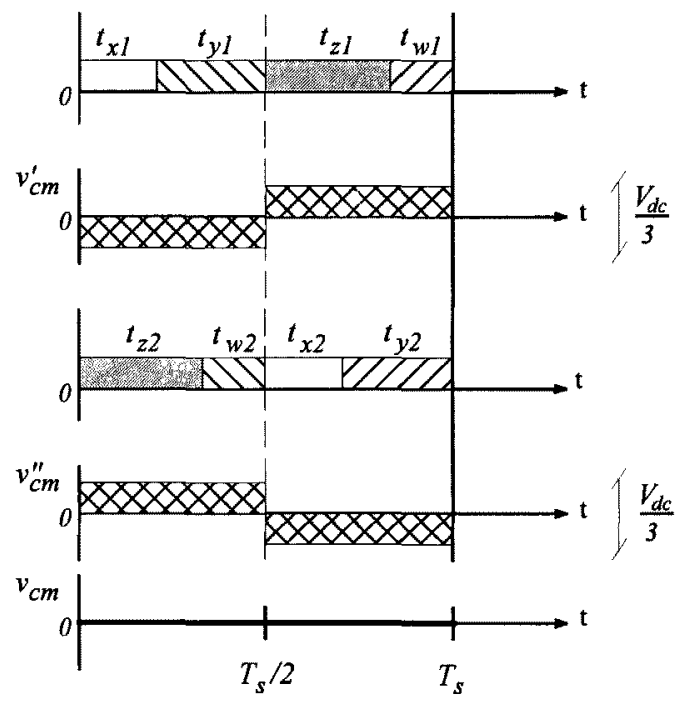

Fig. 3 - Switching times for the two inverters (up) and global common mode voltage $v_{c m}$ (down).

According to Tab. I and eqs. 2 and 3, voltages $v_{c m}^{\prime}$ and $v_{c m}^{\prime \prime}$ are equal to $-V_{D C} / 6$ and $V_{D C} / 6$, respectively. As a result, the condition (5) is fulfilled and the global common mode voltage $v_{c m}$ is zero. Inside the second time segment, two even inverter states $\bar{Z}_{1}$ and $\bar{W}_{1}$ are used in the first inverter and two odd states, $\bar{X}_{2}$ and $\bar{Y}_{2}$, in the second. At $t=T / 2, v_{c m}^{\prime}$ and $v^{\prime \prime}{ }_{c m}$ switch to $\mathrm{V}_{D C} / 6$ and $-\mathrm{V}_{\mathrm{DC}} / 6$, respectively, again satisfying eq. 5 .

Synchronization of common mode voltage variations at $T_{s} / 2$ and synthesis of the two reference voltages are obtained by computing the switching times $t_{x 1}, t_{y 1}, t_{z 1}$, $t_{w 1}, t_{x 2}, t_{y 2}, t_{z 2}, t_{w 2}$ through the following equations:

$$
\begin{gathered}
\bar{V}_{l}^{*} \cdot T_{S}=\bar{V}_{x l} \cdot t_{x l}+\bar{V}_{y 1} \cdot t_{y l}+\bar{V}_{z l} \cdot t_{z l}+\bar{V}_{w 1} \cdot t_{w 1} \\
\bar{V}_{2}^{*} \cdot T_{S}=\bar{V}_{x 2} \cdot t_{x 2}+\bar{V}_{y 2} \cdot t_{y 2}+\bar{V}_{z 2} \cdot t_{z 2}+\bar{V}_{w 2} \cdot t_{w 2} \\
t_{x l}+t_{y l}=T_{S} / 2
\end{gathered}
$$




$$
\begin{aligned}
& t_{z 1}+t_{w 1}=T_{S} / 2 \\
& t_{x 2}+t_{y 2}=T_{S} / 2 \\
& t_{z 2}+t_{w 2}=T_{S} / 2
\end{aligned}
$$

At $t=T / 2$ variations of $v_{c m}^{\prime}$ and $v^{\prime \prime}{ }_{\mathrm{cm}}$ generate common mode current pulses with opposite signs, that flowing through the RLC three phase filters, circulate along the drawn path, without affecting the machines and the ground not generating conducted electromagnetic emissions. Under the hypothesis that the inverters are equipped with power devices having similar dynamic features, the proposed strategy allows also to obtain a full compensation of common mode currents.

In order to hold constant the global common mode voltage in systems composed by three inverters the previous approach must be slightly modified. As shown in Fig. 4, the switching period in the three inverters is divided into three equal time segments.

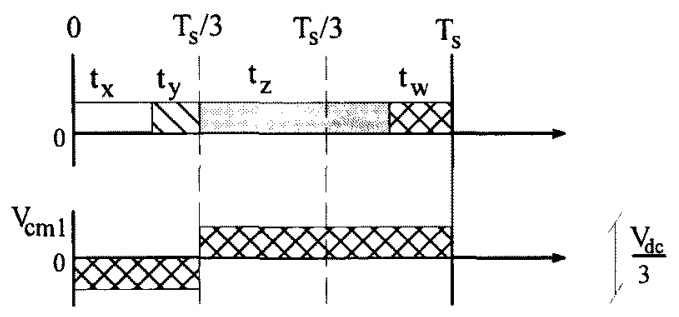

Fig. 4 - Inverter 1 switching time partition.

On inverter 1 along the first time segment two odd states $\overline{\mathrm{X}}_{1}$ and $\overline{\mathrm{Y}}_{1}$ are only used to perform the voltage modulation while two even states $\bar{Z}_{1}$ and $\vec{W}_{1}$ are used in the other two time segments. Accordingly, the common mode voltage switches at $t=0$, from $\mathrm{V}_{\mathrm{DC}} / 3$ to $-\mathrm{V}_{\mathrm{DC}} / 3$ and at $t=T / 3$, from $-\mathrm{V}_{\mathrm{DC}} / 3$ to $\mathrm{V}_{\mathrm{DC}} / 3$.

$$
\begin{gathered}
\bar{V}_{l}^{*} \cdot T_{S}=\bar{V}_{x l} \cdot t_{x l}+\bar{V}_{y l} \cdot t_{y l}+\bar{V}_{z l} \cdot t_{z l}+\bar{V}_{w l} \cdot t_{w l} \\
t_{x 1}+t_{y 1}=T_{S} / 3 \\
t_{x 1}+t_{y 1}+t_{w 1}+t_{z 1}=T_{S}
\end{gathered}
$$

By applying the same modulation technique on the other two inverters, but shifting common mode voltage variations of respectively $T / 3$ and $2 T_{\S} / 3$, as shown in Fig. 5, common mode

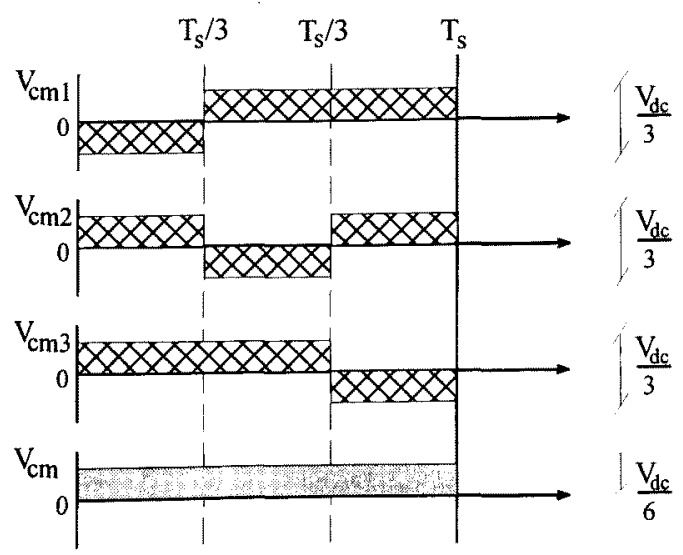

Fig. 5-Global common mode voltage $v_{c m}$. voltage variations are forced to periodically take place with opposite sign in two of the three drives. Consequently, the global common mode voltage still remains constant and the common mode currents are constrained to flow inside two of the three inverters.

RLC three phase filters, shown in Fig. 1, must be suitably designed in order to damp any current circulating between the two inverters, while ensuring an effective short circuit for the high frequency homopolar current components. Parameters of RLC filters used are reported in Tab. II.

\section{TABLE II - PARAMETERS OF RLC FILTERS}

\begin{tabular}{|c|c|}
\hline$R$ & $200 \Omega$ \\
\hline$L$ & $220 \mu \mathrm{H}$ \\
\hline$C$ & $11 \mathrm{nF}$ \\
\hline
\end{tabular}

An alternative to RLC filters consists in connecting the electric machines to the ground by using the same cable. In such a way a six wire system is still obtained, no RLC filters are required but homopolar current components still flow trough the machines, although no ground currents are present.

\section{Experimental Results}

An experimental evaluation of the proposed approach to two and three inverter systems has been carried out. The systems consist of two or three $5 \mathrm{~kW}$ IGBT inverters switching at $10 \mathrm{kHz}$ and connected to the same DC bus. The blanking time has been settled to $1 \mu$ s for all the three inverters, feeding two or three four poles induction machines. Several experimental tests have been performed. In Fig. 6, the measurement scheme of common mode voltages and currents is shown. Firstly, a conventional system with no connection between the two drives and employing a conventional adjacent state Space Vector Modulation (SVM) technique has been considered and the obtained results are shown in Figs. 7-10. The proposed modulation approach has been evaluated for both two inverter and three inverter systems. The obtained results are shown, respectively, in Figs. 11-15 and Figs. 16-21.

As it is possible to observe in Fig. 7, tests have been performed while the two machines are supplied at different frequencies, namely 50 and $25 \mathrm{~Hz}$.

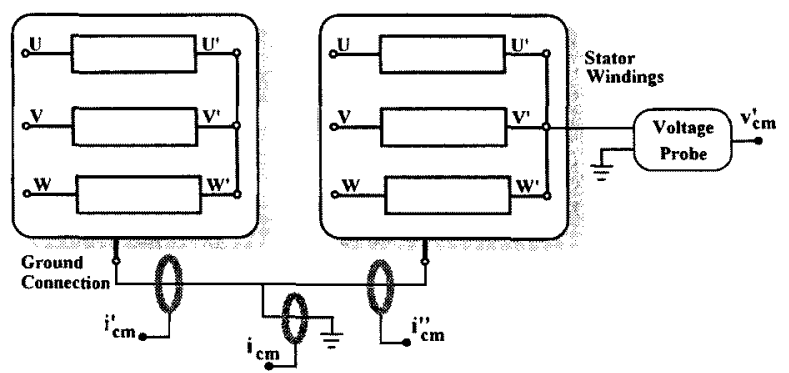

Fig.6 - Measurement scheme of CM voltage and current.

In Fig. 8 and Fig. 9 common mode voltages and ground currents are shown, obtained using an adjacent state SVM technique. In Fig. 10, total ground current spectra are presented, obtained using the SV and the Sine/Triangle modulation techniques.

According to the proposed approach, in Figs. 11 and 12 
common mode voltage are reported, showing that $v_{c m}^{\prime}$ and $v_{c m}^{\prime \prime}$ are opposite, $v_{c m}$ is constantly equal to zero.

The ground current generated by each drive, as well as the total ground current, are shown in Fig. 13. In Fig. 14, a comparison between the total ground current spectra are reported, obtained with the proposed approach, the SV and Sine/Triangle modulation techniques.

The main peaks present in the low frequency range of the spectra considered, depend by the harmonics of the modulations technique and by the reflection phenomena in cables. By adopting the proposed approach ground currents are noticeably lowered. Finally, current flowing through RLC filters are shown in Fig. 15.

In Fig. 16, the phase currents in a three drives system are shown, obtained when the three machines are supplied at $50 \mathrm{~Hz}$. In Fig. 17, $v_{\mathrm{cm}}^{\prime}, v^{\prime \prime} \mathrm{cm}$ and $v^{\prime \prime \prime} \mathrm{cm}$ are shown, while the total common mode voltage $v_{c m}$ is reported in Fig. 18.

Ground currents recorded on the three drive system are shown in Fig. 19, while the currents flowing through RLC filters are reported in Fig. 20. As a comparison, the ground currents obtained using a standard Sine/Triangle modulation technique are shown in Fig. 21. In Fig. 22, a direct comparison among the total ground current spectra is reported, obtained in a three drives system using the proposed approach and a conventional Sine/Triangle modulation technique. As it is possible to observe, the ground current spectrum amplitude performed by the proposed approach is much lower that in the conventional case.

Finally, in Fig. 23 a comparison between the ground current spectra obtained using the proposed approach in two and three drives systems is reported, showing that the proposed approach acts quite similarly.

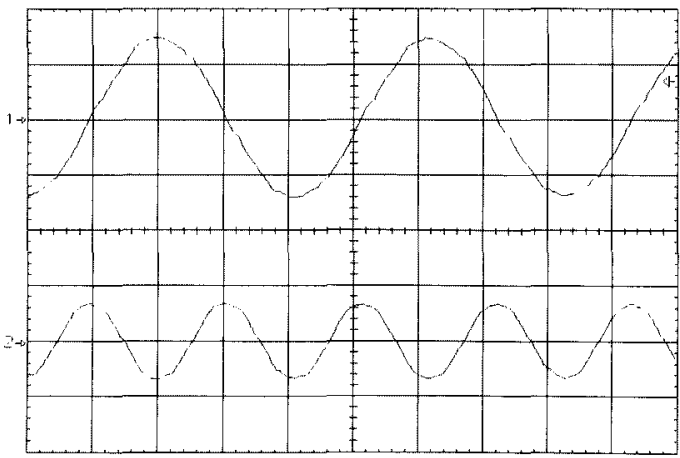

Fig. 7 - Two drives system, SVM. (1) (2) Phase currents in the two drives. Time $10 \mathrm{~ms} / \mathrm{div}$, Current $1 \mathrm{~A} / \mathrm{div}$.

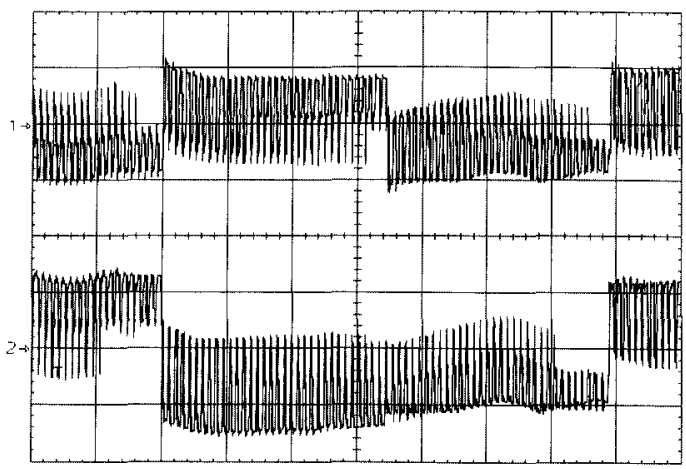

Fig. 8 - Two drives system, SVM. (1),(2) Common mode voltage in the two inverters. Time $1 \mathrm{~ms} / \mathrm{div}$; Voltage $100 \mathrm{~V} / \mathrm{div}$.

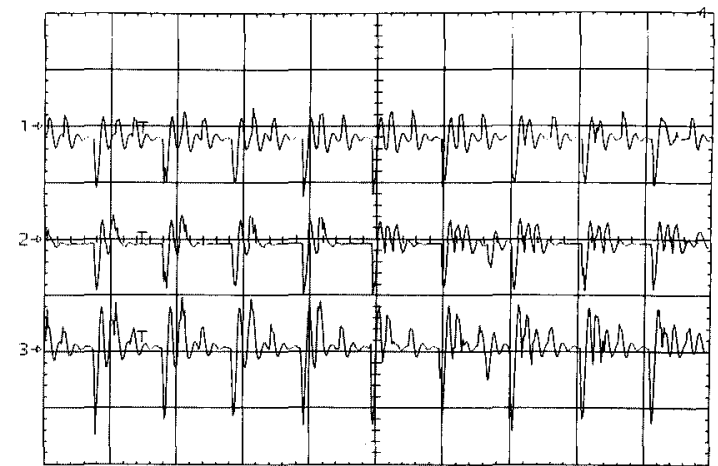

Fig. 9 - Two drives system, SVM. (1), (2) Ground currents in the drives, (3) Total ground current. Time $100 \mathrm{\mu s} / \mathrm{div}$, Current . I A/div.

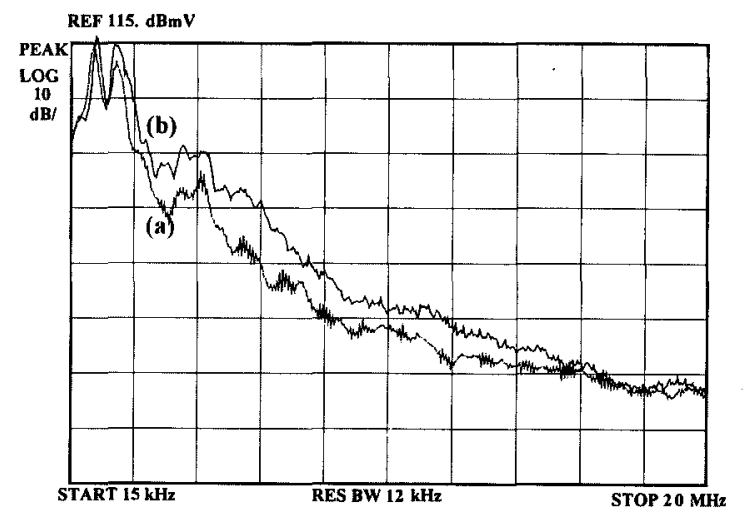

Fig. 10 - Two drives system, (a) Sine/triangle Modulation, (b) SVM. Total ground current frequency spectra.

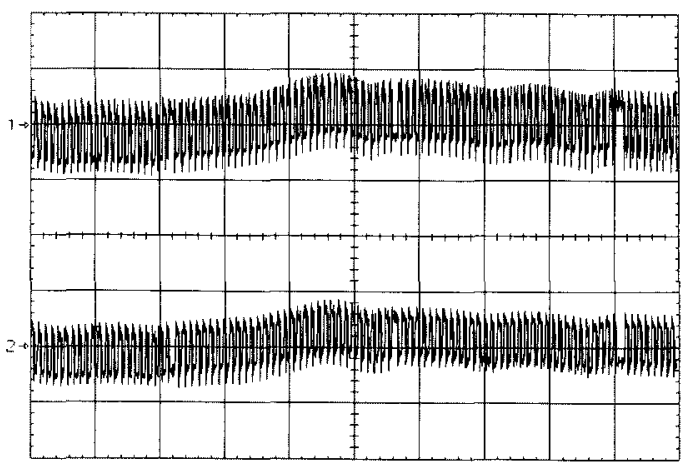

Fig. 11 - Two drives system, proposed approach.(1) $v_{c m}^{\prime}(2)$ $v^{\prime \prime m}$. Time $1 \mathrm{~ms} /$ div; Voltage $100 \mathrm{~V} /$ div.

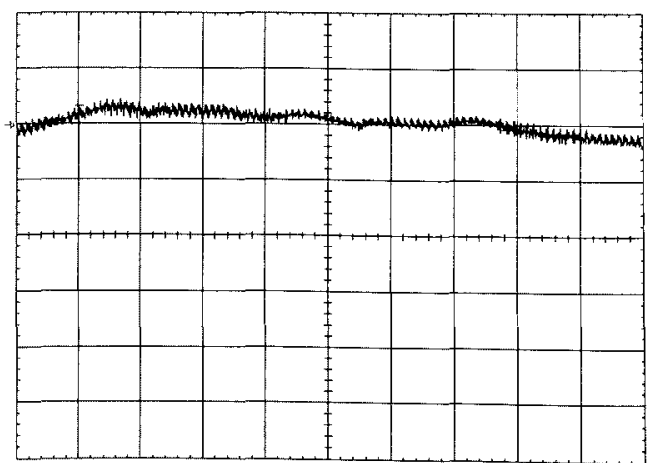

Fig. 12 - Two drives system, proposed approach. (1) $v_{c m}$ Time $1 \mathrm{~ms} /$ div; Voltage 100 V/div. 


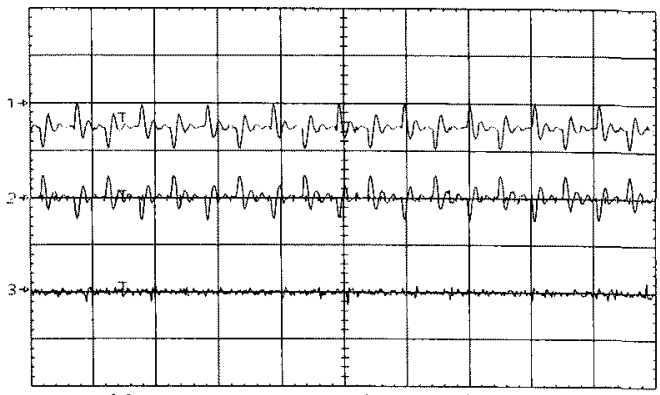

Fig. 13 - Two drives system, proposed approach (1) (2) Drives ground currents, (3) Total ground current. Time $100 \mathrm{\mu} / \mathrm{div}$, Current . $1 \mathrm{~A} / \mathrm{div}$

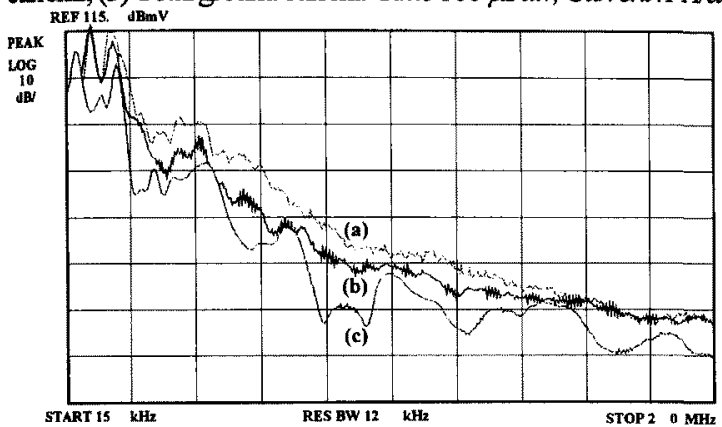

Fig. 14 - Two drives system: (a) Sine/Tri Modulation, (b) SVM, (c) proposed approach. Total ground current spectra.

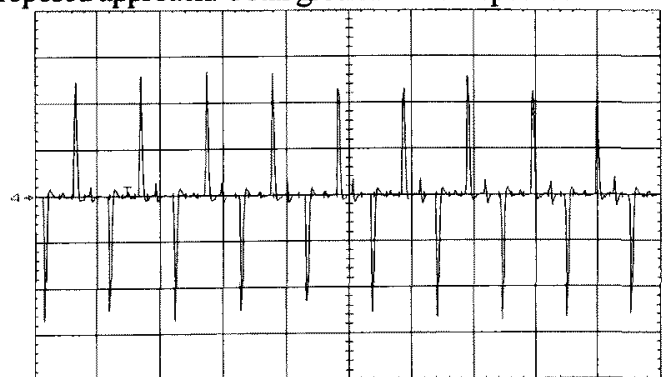

Fig. 15 - Two drives system, proposed approach. (1) Current in RCL filters. Time $100 \mu \mathrm{s} /$ div, Current. I A/div.

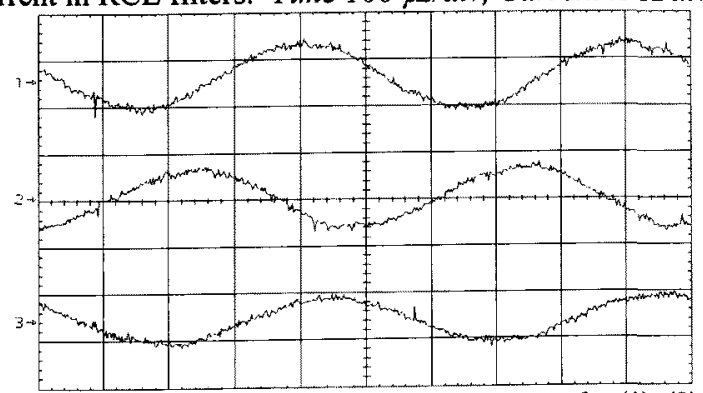

Fig. 16 - Three drives system, proposed approach. (1) (2) (3) Phase current in the three drives. Time $10 \mathrm{~ms} /$ div, Current $1 \mathrm{~A} / \mathrm{div}$.

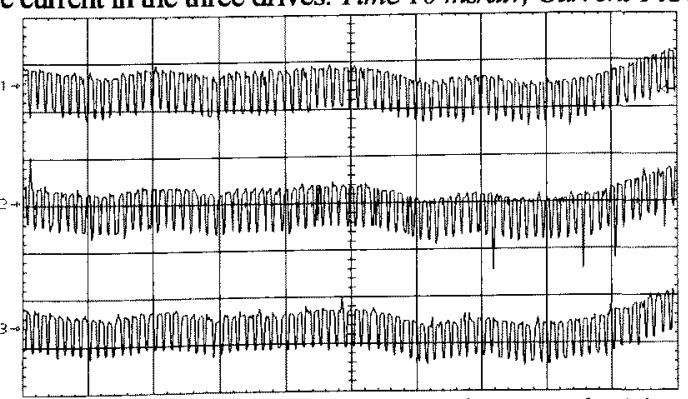

Fig 17 - Three drives system, proposed approach. (1) $v_{\mathrm{cm}}^{\prime}$ (2) $v^{\prime \prime}{ }_{c m},(3) v^{\prime \prime}{ }_{c m}$, Time $1 \mathrm{~ms} /$ div, Voltage $100 \mathrm{~V} / \mathrm{div}$.

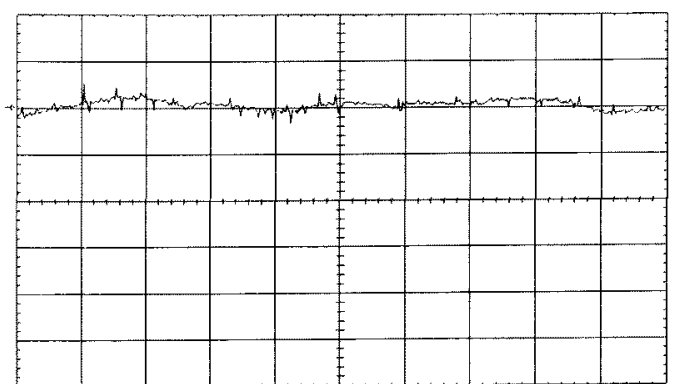

Fig. 18 - Three drives system, proposed approach. (1) $v_{c m}$. Time $1 \mathrm{~ms} /$ div; Voltage 100V/div.

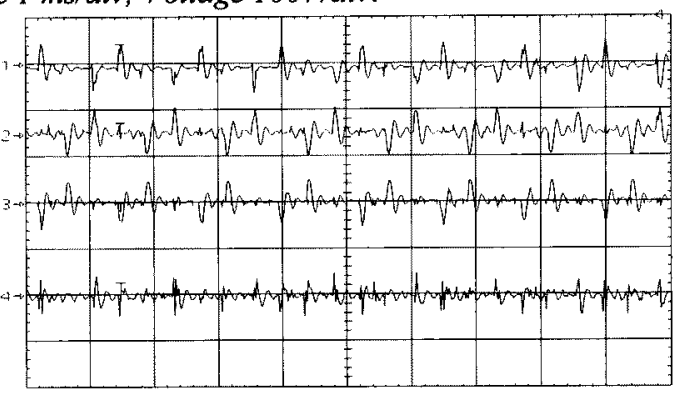

Fig. 19 - Three drives system, proposed approach. (1) (2) (3) Ground currents, (4) Total ground current. Time $100 \mu \mathrm{s} / \mathrm{div}$, Current .1 A/div.

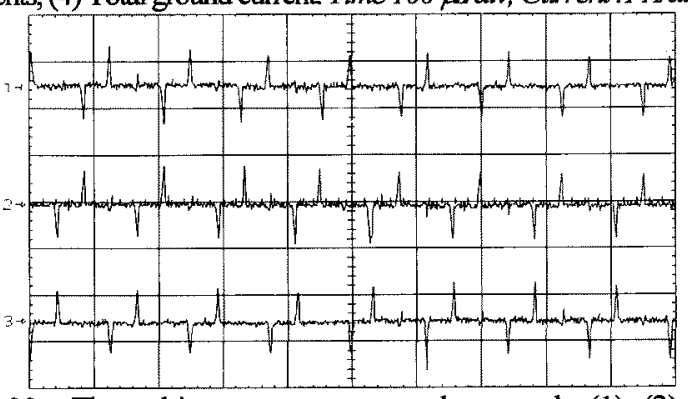

Fig. 20 - Three drives system, proposed approach: (1), (2), (3) Current in RCL filters. Time $100 \mu \mathrm{s} / d \mathrm{dv}$, Current .5 A/div.

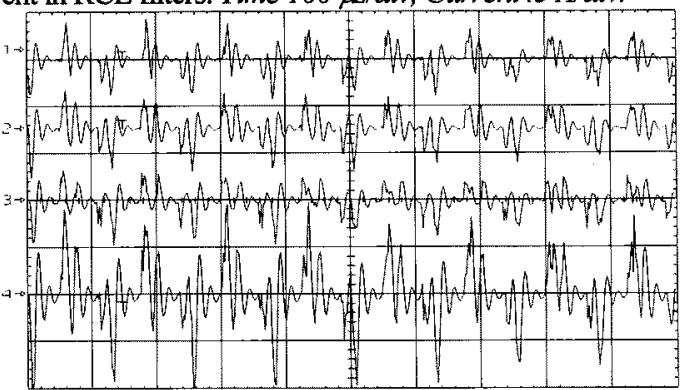

Fig. 21 - Three drives system, Sine/Tri Mod: (1), (2), (3) Drives ground currents, (4) Total ground current. Time $100 \mu \mathrm{s} d \mathrm{div}$, Current . $1 \mathrm{~A} / \mathrm{div}$

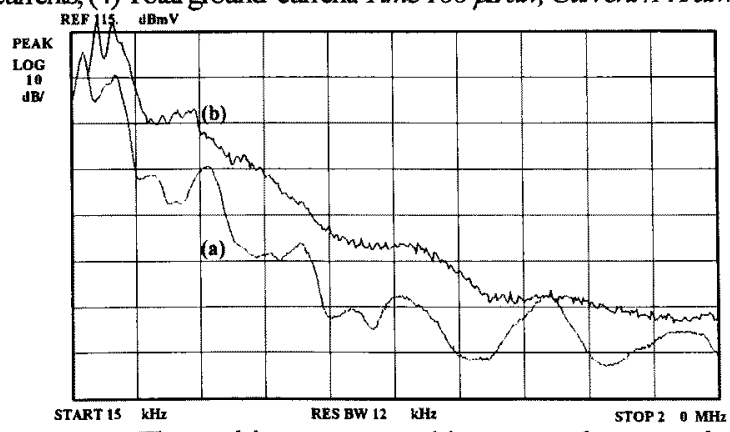

Fig. 22 - Three drives system, (a) proposed approach, (b) Sine/Tri modulation. Total ground current frequency spectrum. 


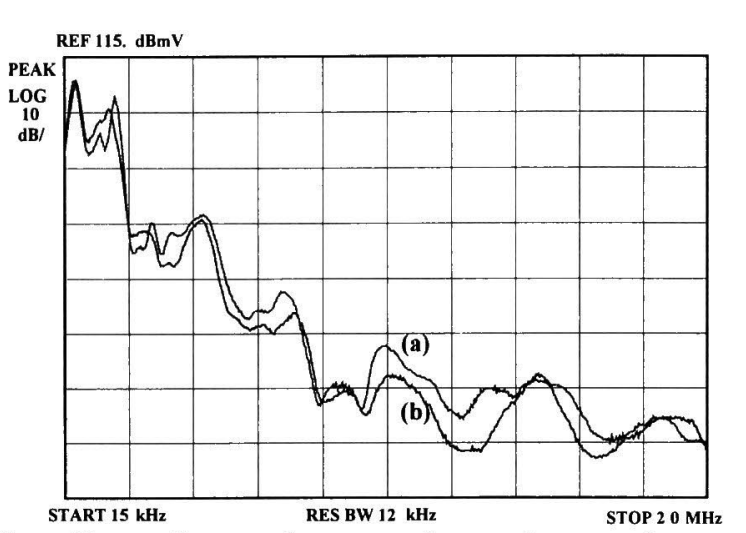

Fig. 23 - Proposed approach, total ground current frequency spectrum: (a) two drives system, (b) three drives system.

\section{Conclusion}

A new common mode current cancellation technique has been presented for two and three drive systems, that represents an inexpensive alternative to conventional common mode filters. It is based on a suitable connection between the inverters and on a new PWM modulation strategy. Such a modulation strategy, manages the inverter state sequences in the inverters in order to synchronize the variation of the common mode voltages. A theoretically zero common mode voltage is then obtained in the multi drive systems, that, thanks to the connection made through RLC filters, can be seen as a n-wire system.

Experimental results confirm the consistence of the proposed approach, showing much lower ground currents than using a conventional approach.

The proposed approach can be easily applied to systems with any number of drives, suitably grouping the drives.

\section{References}

(Manuscript received Jul. 7, 2000, revised Jul. 9, 2001)

[1] S. Ogasawara, H. Akagi, "Modeling and Damping of HighFrequency Leakage Currents in PWM Inverter-Fed AC Motor Drive Systems", IEEE IAS Annual Meeting, October 8-12, 1995, pp. 29-36.

[2] A.Consoli, A. Julian, G.Oriti, A.Testa, "Modeling and Simulation of Common Mode Currents in Inverter Fed Motor Drives", Proceedings of EMC '96 Roma .

[3] T.A. Lipo, S.Chen, E.Zhong, "Improvements in EMI Performance of Inverter-Fed Motor Drives", Proceedings of APEC 1994, March pp.608-614.

[4] M. Cacciato, A. Consoli, G. Scarcella, A. Testa "Reduction of Common Mode Currents in PWM Inverter Motor Drives", IEEE Transactions on Industry Applications, March/April 1999, vol. 35, n.2, pp.469-476.

[5] M. Cacciato, A. Consoli, F. Gennaro, G. Scarcella, A. Testa "Common Mode Current Elimination in Multi-inverter drive Industrial System ", IEEE Industry Application Society Annual Meeting, Phoenix (U.S.A.), 3-7 October 1999, pp. 1851-1857.

[6] G. Oriti, A. Julian, T. Lipo, "Elimination of Common Mode Voltage in Three Phase Sinusoidal Power Converters", IEEE PESC'96, June 23-27, 1996, pp. 1968-1972.

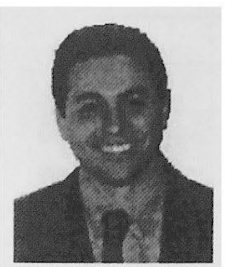

Mario Cacciato was born in Catania, Italy, in 1969. He received the M.S. degree with honor in 1996 in Electrical Engineering from the University of Catania, Italy, and the Ph.D. in Electronic Engineering in 2000 from the University of Reggio Calabria, Italy. Since November 2000 he joined the University of Rome "La Sapienza", Italy, as an assistant professor. He is a Member of IEEE.

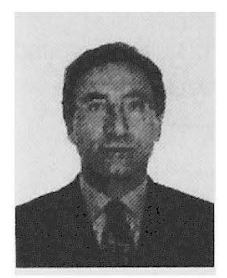

Alfio Consoli was bom in Catania, Italy, in 1949. He graduated in Electrical Engineering from the Politecnico di Torino, Italy. During 1973-1974 he was with FIAT, Torino, Italy. In 1975, he joined the Electrical and Electronic Engineering Department of University of Catania, Catania, Italy, where, since 1985 he has been a Professor in Electrical Engineering, with classes in Electrical Machines and Power Electronics. His research activities include energy conversion systems, electrical drives, robotics, and power electronics. In 1980, he was the recipient of a NATO Grant at the Purdue University, West Lafayette, IN, and in 1985 he was a visiting professor at the University of Wisconsin, Madison, USA.

Professor Consoli is the author of more than 150 technical papers and two international patents. He has also coauthored and coeditored the book "Modern Electric Drives" (Amsterdam, The Netherlands: Kluwer, 2000).

Professor Consoli is a Member of AEI, EPE and a Fellow of IEEE.

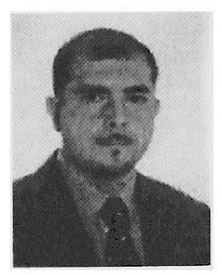

Giuseppe Scarcella was bom in Torino, Italy, in 1969. He received the M.S. degree with honors and the Ph.D. degree from the University of Catania, Catania, Italy, in 1995 and 1999, respectively, both in electrical engineering. During 1995, he was recipient of an SGS-Thomson Grant. He is currently a Researcher in the areas of electrical machines and power electronics at the University of Catania. During 1998, he a was a Visiting Scholar at the University of Wisconsin, Madison. His research interests include sensorless control of electrical drives, digital modulation techniques, and electromagnetic compatibility.

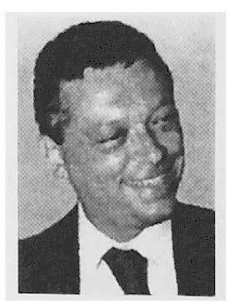

Antonio Testa was bom in Catania, Italy, in 1962. He received the degree in electrical engineering in 1988 from the University of Catania, Italy. During 1989-1990, he was recipient of an SGS-Thomson Grant. From 1990 to 1998, he was an Assistant Professor of Electrical Drives and Power Electronics in the Department of Electrical, Electronic and Systems Engineering, University of Catania. $\mathrm{He}$ is currently a Professor of Electric Machines and Power Electronics at the University of Messina, Sant'Agata, Messina, Italy. During the 1991, he was a Visiting Researcher at the University of Wisconsin, Madison. Professor Testa is coauthor of more than 100 technical papers and two international patents. His major research interest are in the fields of sensorless control of electrical drives, advanced digital control systems, power converters, power devices and electromagnetic compatibility.

Professor Testa is a Member of the IEEE. 